\title{
Tactile working length determination for root canal therapy in underserved settings
}

\section{Abstracted from \\ Jordan RA, Holzner AL, Markovic L, Brueckner I, Zimmer S.}

Clinical effectiveness of basic root canal treatment after 24 months: a randomized controlled trial. J Endod. 2014; 40: 465-470.

Address for correspondence Dr Anna L. Holzner, Dental Clinic 1, Operative Dentistry and Periodontology, University of Erlangen-Nuremberg, Gluckstr 11, D-91054 Erlangen, Germany. E-mail address: aholzner@dent.uni-erlangen.de

\section{Question: Is basic root canal treatment (BRT) with tactile working length determination as effective as standard endodontic treatment after 24 months?}

Design Multicentre randomised controlled clinical trial in a rural underserved African setting.

Intervention The technical and electrical infrastructure still remains unstable in Gambia. Therefore, block randomisation was chosen. Patients included in $\mathbf{2 0 1 0}$ were treated according to the original BRT method with tactile working length determination, whereas patients included in 2011 were treated with radiographic working length control. During the latter study period and the 24-month evaluation, a generator was provided to ensure sufficient power supply to run a radiographic unit.

Outcome measure The primary end point was the apical extension score of the radiographic quality parameter of root canal fillings. The secondary radiographic end point was the periapical index, and the secondary clinical end point was tooth tender to percussion. The safety end point was tooth loss as a consequence of endodontic failure. Results BRT with tactile working length determination compared with standard radiographic working length control did not significantly differ in terms of radiographic and clinical outcomes after 24 months. The apical extension of the root canal fillings and the periapical anatomic structures showed no significant differences according to radiographic analyses $(P=0.5)$. Corresponding results were found in clinical aspects of tooth tender to percussion $(P=0.6)$ and tooth loss $(P=0.7)$.

Conclusions Within the limits of this randomised controlled trial it was shown that tactile working length determination in BRT resulted in comparable treatment outcomes in radiographic and clinical aspects compared with standard endodontic treatment with radiographic working length determination. Tactile working length determination turned out to be an accurate method in BRT.

\section{Commentary}

As the authors reported, caries and tooth loss are still high in underserved populations, particularly in Africa, where about 90\% of the caries burden remains dentally untreated and tooth extraction is still the most common treatment.

The basic root canal treatment (BRT) that the authors investigated is presented by them as an alternative approach that was introduced to primary oral health care in Gambia (West Africa) in 2006.

The technique used in the study does not required the use of a radiograph or any other device to confirm the working length needed to perform root canal treatment in permanent teeth.

The establishment of correct working length is a pivotal step for successful root canal treatment; the epidemiological and histological evidence suggested that minimal contact between canal filling material and periapical tissue produce optimal healing. ${ }^{1,2}$

Although radiography is the standard method of working length determination during root canal treatment, several factors may influence their accuracy such as the shape and orientation of the apex in relation to the X-ray beam, observer variability and film speed. Electronic apex locator proved to be a predictable tool for working length assessment, and it overcomes some shortcomings of radiographs.

New battery-operated apex locators do not required electrical power. Despite having an accuracy of $80-90 \%$ in most root canals, their performance can be limited by multiple factors. Tactile technique using an estimated working length and first binding point of a file is an alternative method for measurement of working length. This method was suggested for relatively straight canals. Several researchers have suggested that a combination of methods increases the accuracy of length determination. ${ }^{3}$

This clinical trial addressed an important topic with a clearly focused question comparing two different techniques to perform root canal treatment in underserved populations, though it's uncertain the use of the block randomisation applied since all patients in 2010 were assigned to an intervention group and all patients in 2011 were assigned to a control group. The settings were different; participants in the test group were treated at the public outpatient dental unit of Kindergarten Wattenscheid in Gambia dental station in Brikama-Kabafita, Western Region, Gambia. The control group was treated, and all follow-up examinations were performed, at the public Swedish Clinic in Kotu, Greater Banjul Area, Gambia.

The authors reported since BRT is a new treatment concept in operative dentistry for underserved communities, a stepwise 
clinical development and proof procedure according to Food and Drug Administration recommendations for pharmacologic drug admission was chosen.

The controlled phase 1 , for procedure safety and tolerance were clinically monitored in 25 cases observationally for 12 months and results were published previously in 2009. The present publication is a controlled phase 2 study to test the treatment concept, in particular tactile working length determination and the clinical and radiographic outcome. The target sample size was predefined with a minimum of 50 cases (n: 25 in the intervention and n: 29 in the control group), sample size was not published in the journal version, yet is clear in the additional information online. ${ }^{4}$

The two groups were not totally similar at the start of the trial, as the test group was mainly males and the control group was mainly females, also the number of posterior teeth in the control group is double the number in the test group.

Although blindness of patients and clinicians to the procedure performed was not possible in this study it is important for the study that the 24-month follow-up in the test and control group was done by an independent investigator who was not part of the operative staff.

The results obtained by the authors have very limited validity and generalisability in endodontic practice in developed countries, however it could be considered in underserved areas where that intervention is the only option instead of extraction.

\section{Abdullah J Dohaithem, Eman 0 Bakarman, Analia Veitz-Keenan NYU College of Dentistry, New York, USA}

1. Ng YL, Mann V, Rahbaran S, Lewsey J, Gulabivala K. Outcome of primary root canal treatment: systematic review of the literature - Part 2. Influence of clinical factors. Int Endod / 2008; 41: 6-31

2. Ricucci D, Langeland K (1998) Apical limit of root canal instrumentation and obturation, part 2. A histological study. Int Endod J 1998; 31: 394-409

3. Kim YJ, Chandler NP. Determination of working length for teeth with wide or immature apices: a review. Int Endod / 2013; 46: 483-491

4. http://www.jendodon.com (http://dx.doi. org/10.1016/j.joen.2013.10.028; 11. 028 Last access online 05/15/2014

Evidence-Based Dentistry (2014), 15, 56-57. doi: 10.1038/sj.ebd.6401029 